\title{
A general Choquet-Deny theorem for nilpotent groups
}

\author{
Albert Raugi \\ IRMAR, université de Rennes I, campus de Beaulieu, 35042 Rennes cedex, France
}

Received 2 December 2002; accepted 10 June 2003

Available online 18 May 2004

\section{Abstract}

Let $G$ be a locally compact second countable nilpotent group. Let $\mu$ be a probability measure on the Borel sets of $G$. We prove that any bounded continuous function $h$ on $G$ solution of the convolution equation

$$
\forall g \in G, \quad \int_{G} h(g x) \mu(d x)=h(g)
$$

verifies $h(g x)=h(g)$ for all $(g, x) \in G \times \operatorname{supp} \mu$.

(C) 2004 Elsevier SAS. All rights reserved.

\section{Résumé}

Soient $G$ un groupe nilpotent localement compact à base séparable et $\mu$ une mesure de probabilité sur les boréliens de $G$. Nous montrons que toute fonction continue bornée $h$ sur $G$ solution de l'équation fonctionnelle

$$
\forall g \in G, \quad \int_{G} h(g x) \mu(d x)=h(g)
$$

admet pour période tous les points du support de $\mu$.

(c) 2004 Elsevier SAS. All rights reserved.

Keywords: Random walk; Harmonic functions

\section{Introduction}

1.1. Let $G$ be a locally compact second countable (lcsc) group with identity element $e$. Let $\mu$ be a probability measure on the Borel- $\sigma$-algebra $\mathcal{B}(G)$ of $G$. A bounded Borel function $h$ on $G$ is called $\mu$-harmonic if it satisfies the mean value property

$$
\forall g \in G, \quad h(g)=\int_{G} h(g x) \mu(d x) .
$$

E-mail address: raugi@univ-rennes1.fr (A. Raugi). 
We denote by $\mathcal{H}_{c}$ the family of bounded continuous $\mu$-harmonic functions. We said that a probability measure $\mu$ is aperiodic if the closed subgroup of $G$ generated by the support of $\mu$ is equal to $G$. To prove the stated result, we are brought to show that, for all aperiodic probability $\mu, \mathcal{H}_{c}$ is reduced to constant functions.

Blackwell [3] has proved the result for a discrete abelian group with a finite number of generators. Later Choquet and Deny [4] have extended it to all abelian groups.

A significant improvement of this result, with a very simple proof, is the following. Let $(S,+)$ be an algebraic abelian semi-group equipped with a $\sigma$-algebra $\mathcal{S}$. We assume that the application from $S \times S$ to $S$ which sends $(x, y)$ to $x+y$ is measurable. Let $\mu$ be a probability measure on $\mathcal{S}$. Then any bounded $\mu$-harmonic function $h$ satisfies: for all $x \in S$, for $\mu$-almost every $y \in S, h(x+y)=h(x)$. (Consider the sequence of functions defined by:

$$
u_{1}(x)=\int_{S}\left(h\left(x+y_{1}\right)-h(x)\right)^{2} \mu\left(d y_{1}\right)
$$

and for all $n \geqslant 2$,

$$
u_{n}(x)=\int_{S}\left(h\left(x+y_{1}+\cdots+y_{n}\right)-h\left(x+y_{2}+\cdots+y_{n}\right)\right)^{2} \mu\left(d y_{1}\right) \ldots \mu\left(d y_{n}\right) .
$$

One easily sees that the sequence $\left(u_{n}\right)_{n \geqslant 0}$ is increasing (Cauchy-Schwarz theorem) and, for all $x \in S$, the series $\sum_{n \geqslant 0} u_{n}(x)$ is a telescopic convergent series $\left(u_{n}(x)=\int_{S} h^{2}(x+y) \mu^{n}(d y)-\int_{S} h^{2}(x+y) \mu^{n-1}(d y)\right)$. Hence the result.)

Dynkin and Malyutov [6] have proved that $\mathcal{H}_{c}$ is reduced to the constant functions for a discrete nilpotent group with a finite number of generators. Azencott [1] has obtained this result for a class of groups, containing the connected nilpotent groups, when $\mu$ is a spread out probability (i.e. there exists an integer $n \geqslant 1$ such that the $n$-fold convolution $\mu^{* n}$ of $\mu$ by itself is nonsingular with respect to a Haar measure on $G$ ). In [15] it is pointed out that, in the work of Azencott, the spread out assumption is only necessary for ensuring that any bounded $\mu$-harmonic function is right uniformly continuous and Azencott's result extends to general $\mu$ if we restrict our study to bounded right continuous $\mu$-harmonic functions. Guivarc' $h$ [9] has proved the result for a class of groups, containing nilpotent groups, for which there exists a compact neighborhood $V$ of the identity generating $G$ such that the series $\sum n^{a} \mu\left(V^{n+1}-V^{n}\right)$ converges for an $a>0$. He also obtained the result for general aperiodic probability $\mu$ when $G$ is a nilpotent group of order two. Avez [2] has showed the result for a group with nonexponential growth carrying an aperiodic probability with finite support. Other references on this subject, for other types of groups, are $([5,8,10-13,16])$.

In this paper we are proving the result for a nilpotent group and for a general aperiodic probability $\mu$.

\section{Bounded harmonic function on nilpotent groups}

2.1. Theorem. Let $G$ be a nilpotent locally compact second countable group. Let $\mu$ be an aperiodic probability measure on $G$. Then any continuous bounded $\mu$-harmonic function on $G$ is constant.

The remaining of this section is devoted to the demonstration of this theorem.

\section{Preliminaries}

2.2. Let $m$ be a right Haar measure on $G$. For all $f \in \mathbb{L}^{\infty}(G, m)$ and all $\alpha \in \mathbb{L}^{1}(G, m)$ the function $f^{\alpha}: g \rightarrow$ $\int_{G} f(x g) \alpha(x) m(d x)$ is left uniformly continuous (l.u.c.) on $G$. That is,

$$
\sup _{x \in G}\left|f^{\alpha}(g x)-f^{\alpha}(x)\right| \leqslant\|f\|_{\mathbb{L}^{\infty}(G, m)}\left\|\alpha\left(\cdot g^{-1}\right)-\alpha(\cdot)\right\|_{\mathbb{L}^{1}(G, m)},
$$


and $\delta(g)=\left\|\alpha\left(\cdot g^{-1}\right)-\alpha(\cdot)\right\|_{\mathbb{L}^{1}(G, m)}$ is a continuous function on $G$ satisfying $\delta(e)=0 . f^{\alpha}$ is $\mu$-harmonic when $f$ is. Let $\left(\alpha_{n}\right)_{n \geqslant 0}$ be a sequence of functions on $G$ forming an approximate identity in $\mathbb{L}^{1}(G, m)$. That is, for all $n \geqslant 0, \alpha_{n}$ is a continuous non-negative function, satisfying $\int_{G} \alpha_{n}(x) m(d x)=1$ and with a support contained in a compact neighborhood $V_{n}$ of $e$ such that $\bigcap_{n \geqslant 0} V_{n}=\{e\}$. Then, for all continuous and bounded function $f$ on $G, f^{\alpha_{n}}$ converges uniformly on compact sets to $f$. We shall denote by $\mathcal{H}_{\text {l.u.c. }}$ the family of all l.u.c. $\mu$-harmonic function on $G$. To show the theorem it is enough to prove that any element of $\mathcal{H}_{\text {l.u.c. }}$. is constant.

2.3. We denote by $\lambda$ the probability measure $\sum_{k \geqslant 0} 2^{-(k+1)} \mu^{* k}$. Any $\mu$-harmonic function is also a $\lambda$-harmonic function. The support $\operatorname{supp} \lambda$ of the probability $\lambda$ is equal to the closure $T_{\mu}$ of the semigroup $\bigcup_{k \in \mathbb{N}}(\operatorname{supp} \mu)^{k}$. Replacing, if necessary, $\mu$ by $\lambda$ we can suppose that the support of $\mu$ is the semigroup $T_{\mu}$ of $G$, all the convolutions $\mu^{k *}, k \geqslant 1$, are equivalent measures and $\mu^{k *}(\{e\})>0$.

If $H_{1}$ and $H_{2}$ are two subgroups of $G$ we denote by $\left[H_{1}, H_{2}\right]$ the closed subgroup of $G$ generated by the commutators $[x, y]=x y x^{-1} y^{-1},(x, y) \in H_{1} \times H_{2}$. We call $r$ the nilpotence order of $G$ and we denote by

$$
G_{0}=G \supset G_{1}=[G, G] \supset \cdots \supset G_{r+1}=\left[G, G_{r}\right]=\{e\}
$$

the lower central series of $G$. For all $q \in\{1, \ldots, r+1\}$, we call $\pi_{q}$ the natural map from $G$ onto the quotient group $G / G_{q}$.

2.4. By the right random walk of law $\mu$ we mean the sequence of products

$$
X_{0}=e \quad \text { and } \quad \forall n \geqslant 1, X_{n}=Y_{1} \cdots Y_{n} ;
$$

where $\left(Y_{n}\right)_{n \geqslant 1}$ is a sequence of i.i.d. $G$-valued random variables, defined on a probability space $(\Omega, \mathcal{F}, \mathbb{P})$, whose common distribution is $\mu$.

\section{Proof of Theorem 2.1}

Let $h \in \mathcal{H}_{\text {l.u.c. }}$. We denote by $H$ the period group of $h$; i.e. the closed normal subgroup of $G$ defined by:

$$
H=\left\{g \in G: \forall(x, y) \in G^{2}: h(x g y)=h(x y)\right\} .
$$

We reason ad absurdum, assuming that $H$ is not equal to $G$.

If $\pi$ is the natural map from $G$ onto $G / H$, we have $h=\tilde{h} \circ \pi$ for a left uniformly continuous $\pi(\mu)$-harmonic function on $G / H$. Replacing the triplet $(G, \mu, h)$ by $(G / H, \pi(\mu), \tilde{h})$ we can suppose that $H=\{e\}$.

First stage. We will need the following important lemma.

2.5. Lemma. Let $H$ be a closed normal subgroup of $G$ and $\pi$ the natural map from $G$ onto $G / H$. Let $\mu$ be a probability measure on the Borel sets of $G$. The following assertions are equivalent:

(i) There exists a Borel subset $W$ of $G^{2}$ with $\mu \otimes \mu(W)=1$ such that for $\mu$-almost every $u,(u, u)$ belongs to $W$ and for all $(u, v) \in W$ verifying $\pi(u)=\pi(v)$, we have $u=v$.

(ii) There exists a Borel map $s: G / H \rightarrow G$ such that for $\mu$-almost every $u, u=s \circ \pi(u)$.

(iii) There exists a Borel subset $V$ of $G$ of $\mu$-measure 1 such that for all $(u, v) \in V^{2}$ verifying $\pi(u)=\pi(v)$ we have $u=v$.

Proof. The only nonobvious implication is (i) $\Rightarrow$ (ii).

From hypothesis on $W$, it follows that there exists a Borel set $V$ with $\mu(V)=1$ such that for any $u \in V$ there exists a Borel set $W_{u}$ of $\mu$-measure 1 , such that $u \in W_{u},\{u\} \times W_{u} \subset W$ and $W_{u} \times\{u\} \subset W$. 
For all $u \in G$ we denote by $\bar{u}$ the element $\pi(u)$ of $G / H$. We consider a disintegration of $\mu$ along the classes modulo $H$. For all bounded Borel functions $f$ on $G$ and $g$ on $G / H$, we can write,

(i) $\int_{G} f(u) g(\bar{u}) \mu(d u)=\int_{G} \operatorname{Pf}(\bar{u}) g(\bar{u}) \mu(d u)$,

where $P$ is a transition probability from $G / H$ to $G$ (i.e. an application from $G / H \times \mathcal{B}(G)$ to $[0,1]$ satisfying the two following conditions

(ii) for all $\bar{u} \in G / H, P(\bar{u}, \cdot)$ is a probability measure on the Borel sets of $G$,

(iii) for all Borel set $B$ of $G$, the map $\bar{u} \rightarrow P(\bar{u}, B)$ is $\mathcal{B}(G / H)$-measurable.)

We choose a sequence $\left(\mathcal{P}_{n}\right)_{n \geqslant 0}$ of Borel countable partitions of $G / H$ such that: each member of $\mathcal{P}_{1}$ is bounded; for all $n \in \mathbb{N}$, each member of $\mathcal{P}_{n}$ is the union of some subfamily of $\mathcal{P}_{n+1}$ and

$$
\lim _{n \rightarrow+\infty} \sup \left\{\operatorname{diam}(A): A \in \mathcal{P}_{n}\right\}=0 .
$$

If $g$ is a bounded Borel function on $G / H$, we know [7, Theorems 2.8.19 and 2.9.8] that for all $\pi(\mu)$-almost every $y \in G / H, \forall n \in \mathbb{N}, \pi(\mu)\left(A_{n, y}\right)>0$ and

$$
\lim _{n \rightarrow+\infty}\left(\int_{A_{n, y}} g(x) \pi(\mu)(d x) / \pi(\mu)\left(A_{n, y}\right)\right)=g(y) ;
$$

where, for all $n \in \mathbb{N}, A_{n, y}$ is the member of the partition $\mathcal{P}_{n}$ containing $y$. (Remark that the $\sigma$-algebras generated by the partitions are increasing. If we know that the union of these $\sigma$-algebras generate the Borel $\sigma$-algebra, this result is a consequence of the convergence $\mu$-a.e of the martingale $\left(\mathbb{E}_{\mu}\left[f \mid \sigma\left(\mathcal{P}_{n}\right)\right]\right)_{n \in \mathbb{N}}$ to $\mathbb{E}_{\mu}[f \mid \mathcal{B}(G)]=f$.)

Let $f$ be a non-negative function of $C_{K}(G)$, the space of continuous function on $G$ with compact support. We consider the Borel subset $U$, with $\mu(U)=1$, defined by:

$$
U=\left\{u \in V: \forall n \in \mathbb{N}, \pi(\mu)\left(A_{n, \bar{u}}\right)>0 \text { and } \lim _{n \rightarrow+\infty}\left(\int_{A_{n, \bar{u}}} \operatorname{Pf}(x) \pi(\mu)(d x) / \pi(\mu)\left(A_{n, \bar{u}}\right)\right)=\operatorname{Pf}(\bar{u})\right\} .
$$

Let $u_{0} \in U$ and $\varepsilon>0$. As any probability measure on a polish topological space is regular [14, Proposition II-7-3] and the Borel subsets $A_{n, \bar{u}_{0}}, n \in \mathbb{N}$, are decreasing, we can find a decreasing sequence $\left(K_{n}\right)_{n \geqslant 0}$ of compact subsets of $G$ such that $K_{n} \subset \pi^{-1}\left(A_{n, \bar{u}_{0}}\right) \cap W_{u_{0}}$ and $\mu\left(K_{n}\right) \geqslant(1-\varepsilon) \pi(\mu)\left(A_{n, \bar{u}_{0}}\right)$. Then we have:

$$
\int_{G} f(u) 1_{A_{n, \bar{u}_{0}}}(\bar{u}) \mu(d u) / \pi(\mu)\left(A_{n, \bar{u}_{0}}\right) \geqslant(1-\varepsilon) \int_{G} f(u) 1_{K_{n}}(u) \mu(d u) / \mu\left(K_{n}\right) .
$$

However $\bigcap_{n \in \mathbb{N}} K_{n}=\left\{u_{0}\right\}$ (if $x$ belongs to $\bigcap_{n \in \mathbb{N}} K_{n}$, then $\bar{x}=\bar{u}_{0}$ and $x \in K_{0} \subset W_{u_{0}}$, thus $x=u_{0}$ ). For all open balls $B\left(u_{0}, r\right)$ of center $u_{0}$ and radius $r>0$, it follows that $\bigcap_{n \in \mathbb{N}}\left(K_{n} \cap B\left(u_{0}, r\right)^{c}\right)=\emptyset$ and there exists $p \in \mathbb{N}$ such that $K_{p} \cap B\left(u_{0}, r\right)^{c}=\emptyset$. We deduce that, for all $r>0$,

$$
\lim _{n \rightarrow+\infty} \int_{B\left(u_{0}, r\right)^{c}} 1_{K_{n}}(u) \mu(d u) / \mu\left(K_{n}\right)=0
$$

and therefore

$$
\lim _{n \rightarrow+\infty} \int_{G} f(u) 1_{K_{n}}(u) \mu(d u) / \mu\left(K_{n}\right)=f\left(u_{0}\right) .
$$

By what precedes, it results that for all $u_{0} \in U, f\left(u_{0}\right) \leqslant P f\left(\bar{u}_{0}\right)$. As $\int_{G} f(u) \mu(d u)=\int_{G} P f(\bar{u}) \mu(d u)$ and $C_{K}(G)$ is separable for the uniform norm, it follows that, there exists a Borel set $X$ with $\mu(X)=1$ such that, for 
all $u \in X: \forall f \in C_{K}(G), f(u)=P f(\bar{u})$ and therefore $P(\bar{u}, \cdot)=\delta_{u}$. One can assume that $X$ is a countable union of compact subsets; then $\pi(X)$ is a Borel set of $G / H$. For any $y \in \pi(X)$ denote by $s(y)$ the support of the Dirac measure $P(y, \cdot)$. For all Borel subsets $B$ of $G$, we have

$$
\pi(X) \cap s^{-1}(B)=\pi(X) \cap\left\{1_{B} \circ s=1\right\}=\pi(X) \cap\left\{P 1_{B}=1\right\}
$$

which shows that $s$ is a Borel map from $X$ to $G$. We extend $s$ to a Borel map from $G / H$ to $G$.

From equalities (i) above, it follows that on one hand, for $\pi(\mu)$-almost every $y \in G / H, y=\pi(s(y))$ and on the other hand $s(\pi(\mu))=\mu$. Consequently, for $\mu$-almost all $u \in G, u=s(\pi(u))$.

Second stage. By downward induction we prove that for all $q \in\{1, \ldots, r+1\}$ there exists a Borel subset $V_{q}$ of $G$ with $\mu\left(V_{q}\right)=1$ such that $V_{q} V_{q}^{-1} \cap G_{q}=\{e\}$.

For $q=r+1$, we can take $V_{r+1}=G$. Let us assume the result true for some $q \in\{2, \ldots, r+1\}$. Consider the right random walk $\left(X_{n}\right)_{n \geqslant 0}$ on $G$. We denote by $\mathcal{F}_{0}$ the trivial $\sigma$-algebra $\{\emptyset, \Omega\}$ and, for all $n \geqslant 1, \mathcal{F}_{n}$ the $\sigma$-algebra generated by the random variables $Y_{1}, \ldots, Y_{n}$. For all $g \in G$, the sequence of random variables $\left(h\left(g X_{n}\right)\right)_{n \geqslant 0}$ is a bounded martingale with respect to the filtration $\left(\mathcal{F}_{n}\right)_{n \geqslant 0}$. Therefore it converges $\mathbb{P}$-a.e. and in norm $\mathbb{L}^{s}(\Omega, \mathcal{F}, \mathbb{P})$, for all $s \in[1,+\infty[$;

$$
\forall n \geqslant 0, \forall g \in G, \quad h(g)=\mathbb{E}\left[h\left(g X_{n}\right)\right]=\mathbb{E}\left[\lim _{p \rightarrow+\infty} h\left(g X_{p}\right)\right] .
$$

Moreover we have:

$$
\sum_{n \in \mathbb{N}} \int_{T_{\mu}} \mathbb{E}\left[\left(h\left(g X_{n} x\right)-h\left(g X_{n}\right)\right)^{2}\right] \mu(d x) \leqslant \sum_{n \in \mathbb{N}}\left(\mathbb{E}\left[h^{2}\left(g X_{n+1}\right)\right]-\mathbb{E}\left[h^{2}\left(g X_{n}\right)\right]\right) \leqslant\|h\|_{\infty}^{2}<+\infty .
$$

It follows that for all $g \in G$ and for $\mu$-almost every $x \in G$, the sequences $\left(h\left(g X_{n}(\omega) x\right)\right)_{n} \geqslant 0$ and $\left(h\left(g X_{n}(\omega)\right)\right)_{n} \geqslant 0$ converge, for $\mathbb{P}$-a.e. $\omega \in \Omega$, to the same limit.

As $h$ is left uniformly continuous, we deduce that there exists a Borel set $U$, with $\mu(U)=1$, such that, for all $(g, x) \in G \times U$, the sequences $\left(h\left(g X_{n}(\omega) x\right)\right)_{n \geqslant 0}$ and $\left(h\left(g X_{n}(\omega)\right)\right)_{n \geqslant 0}$ converge, for $\mathbb{P}$-a.e. $\omega \in \Omega$, to the same limit.

Now let us define:

$$
W=\left\{(u, v) \in U \times U \text { : for } \mu \text {-almost all } x \in G, u x v(v x u)^{-1} \in V_{q} V_{q}^{-1}\right\} .
$$

As $\mu^{3}$ and $\mu$ are equivalent measures, the Borel subset $W$ of $G^{2}$ has $\mu \otimes \mu$-measure 1 . In the same way, for $\mu$-almost all $u \in V_{q},(u, u) \in W$ and for all $(u, v) \in W$, for $\mu$-almost all $t,(u t, v t) \in W$.

For $(u, v) \in W$ such that $\pi_{q-1}(u)=\pi_{q-1}(v)$, we have $\mathbb{P}$-a.e.,

$$
\begin{aligned}
\lim _{n} h\left(u X_{n}\right) & =\lim _{n} h\left(u X_{n} v\right)=\lim _{n} h\left(\left(u X_{n} v\right)\left(v X_{n} u\right)^{-1} v X_{n} u\right) \\
& =\lim _{n} h\left(v X_{n} u\right)=\lim _{n} h\left(v X_{n}\right),
\end{aligned}
$$

because $\left(u X_{n} v\right)\left(v X_{n} u\right)^{-1}=\left[u v^{-1}, v X_{n}\right] \in V_{q} V_{q}^{-1} \cap G_{q}=\{e\}$ and therefore

$$
h(u)=\mathbb{E}\left[\lim _{n} h\left(u X_{n}\right)\right]=\mathbb{E}\left[\lim _{n} h\left(v X_{n}\right)\right]=h(v) .
$$

From the continuity of $h$ and the last property of $W$, it follows that

$$
\forall(u, v) \in W \text { such that } \pi_{q-1}(u)=\pi_{q-1}(v), \forall t \in T_{\mu}, h(u t)=h(v t) .
$$

Applying this result to the left translates $h^{g}: x \rightarrow h(g x)(g \in G)$ of $h$, we obtain

$$
\forall g \in G, \forall(u, v) \in W \text { such that } \pi_{q-1}(u)=\pi_{q-1}(v), \forall t \in T_{\mu}, h(g u t)=h(g v t) .
$$


Now setting $H=\left\{x \in G: \forall g \in G, \forall t \in T_{\mu}, h(g x t)=h(g t)\right\}$, we obtain a closed subgroup of $G$. From next Lemma 2.6, it follows that $H$ is a normal subgroup of $G$ contained in the period group of $h$. Consequently $u^{-1} v$ is a period of $h$ and $u=v$. Then, from Lemma 2.5, we deduce that there exists a Borel subset $V_{q-1}$ of $G$, with $\mu\left(V_{q-1}\right)=1$ and $V_{q-1} V_{q-1}^{-1} \cap G_{q-1}=\{e\}$.

Last stage. Finally, for $q=1$, we obtain a Borel subset $V_{1}$ with $\mu\left(V_{1}\right)=1$ such that $V_{1} V_{1}^{-1}=e$. It follows that $T_{\mu} T_{\mu}^{-1}=e$. However $T_{\mu} T_{\mu}^{-1}$ is a subgroup [1, Lemme IV 11] dense in $G$ because $\mu$ is aperiodic, hence $G=\{e\}$ and we end to a contradiction.

2.6. Lemma. Let $G$ be a lcsc nilpotent group and $H$ a subgroup of $G$. For all $t \in G$,

$$
t^{-1} H t \subset H \quad \Rightarrow \quad t^{-1} H t=H .
$$

Proof. We proceed by induction on the order of nilpotence $r$ of $G$. If $r=1$ the property is trivial. Assume the property true for a nilpotent group of order $r \geqslant 1$ and let $G$ a nilpotent group of order $r+1$.

We take again the notations of Section 2.3 and note $\pi$ instead of $\pi_{r+1}$. We have $\pi(t)^{-1} \pi(H) \pi(t) \subset \pi(H)$. However $\pi(H)$ is a closed subgroup of the nilpotent group $G / G_{r+1}$ of order $r$. Therefore $\pi(t)^{-1} \pi(H) \pi(t)=$ $\pi(H)$.

Consequently, if $y \in H$, then there exists $x \in H$ such that $\pi(y)=\pi\left(t^{-1} x t\right)$. It follows that $z=y^{-1} t^{-1} x t$ belongs to $G_{r+1} \cap H$ and therefore $y=t^{-1} x t z^{-1}=t^{-1} x z^{-1} t$. Hence the result.

\section{Other result}

3.1. Definitions. An element $g$ of $G$ is said recurrent if for all neighborhood $V$ of $g, \sum_{n \geqslant 0} 1_{V}\left(X_{n}\right)=+\infty$, $\mathbb{P}$-a.e.. If $g$ is recurrent then, for $\mathbb{P}$-almost all $\omega \in \Omega$, the sequence $\left(X_{n}(\omega)\right)_{n} \geqslant 0$ admits $g$ as a closure value. It is well-known that (see for example [17, Chapter $3, \S 4]$ ):

(i) If one element of $G$ is recurrent then all the elements of $G$ are recurrent. In this case we say that the random walk $\left(X_{n}\right)_{n \geqslant 0}$ on $G$ is recurrent. In the contrary case, we say that the random walk $\left(X_{n}\right)_{n \geqslant 0}$ on $G$ is transient.

(ii) The random walk $\left(X_{n}\right)_{n \geqslant 0}$ on $G$ is recurrent if and only if $\sum_{n \geqslant 0} \mu^{* n}(V)=+\infty$ for all nonempty open set $V$.

(iii) The random walk $\left(X_{n}\right)_{n \geqslant 0}$ on $G$ is transient if and only if $\sum_{n \geqslant 0} \mu^{* n}(V)<+\infty$ for all relatively compact open set $V$.

Let $H$ be a lcsc group on which $G$ acts by automorphisms. For all $g \in G$, we denote by $A(g)$ the automorphism of $H$ associated to $g$. We call $\bar{A}(G)$ the closure of the subgroup $A(G)=\{A(g): g \in G\}$ in the group $\operatorname{Aut}(H)$ of all automorphisms of $H$. We shall say that the action of the pair $(G, \mu)$ on $H$ is recurrent if the random walk $\left(A\left(X_{n}\right)\right)_{n \geqslant 0}$ is recurrent in $\bar{A}(G)$. In other words, for all $u \in H$, the set of closure values of the sequence $\left(A\left(X_{n}\right) u\right)_{n} \geqslant 0$ is the closure of $A(G) u, \mathbb{P}$-a.e.

3.2. Theorem. Suppose that the pair $(G, \mu)$ acts, by interior automorphisms, in a recurrent way on the subgroup $H=[G, G]$. Then $\mathcal{H}_{c}$ is reduce to constants.

Proof. It is enough to prove the result for $h \in \mathcal{H}_{\text {l.u.c. }}$. We denote by $\pi$ the natural map from $G$ to $G / H$. We consider the Borel set $U$ of second stage of the proof of Theorem 2.1. For all $(g, x) \in G \times U$, the sequences $\left(h\left(g X_{n}(\omega) x\right)\right)_{n \geqslant 0}$ and $\left(h\left(g X_{n}(\omega)\right)\right)_{n} \geqslant 0$ converge, for $\mathbb{P}$-a.e. $\omega \in \Omega$, to the same limit. For $u, v \in U$ such that $\pi(u)=\pi(v)$, we have, $\mathbb{P}$-a.e., 


$$
\begin{aligned}
\lim _{n} h\left(u X_{n}\right) & =\lim _{n} h\left(u X_{n} v\right)=\lim _{n} h\left(u X_{n} v u^{-1} u\right) \\
& =\lim _{n} h\left(\left(u X_{n} v u^{-1} X_{n}^{-1} u^{-1}\right) u X_{n} u\right)=\lim _{n} h\left(v X_{n} u\right)=\lim _{n} h\left(v X_{n}\right) .
\end{aligned}
$$

(Remark that $\left(u X_{n} v u^{-1} X_{n}^{-1} u^{-1}\right) u X_{n} u$ and $v X_{n}$ are two elements of $G$ such that

$$
\left|h\left(\left(u X_{n} v u^{-1} X_{n}^{-1} u^{-1}\right) u X_{n} u\right)-h\left(v X_{n} u\right)\right| \leqslant\|h\|_{\infty} \delta\left(\left(u X_{n} v u^{-1} X_{n}^{-1} u^{-1}\right) u v^{-1}\right)
$$

that converges to zero along a subsequence.)

It follows that for all $(u, v) \in U^{2}$ verifying $\pi(u)=\pi(v)$,

$$
h(u)=\mathbb{E}\left[\lim _{n} h\left(u X_{n}\right)\right]=\mathbb{E}\left[\lim _{n} h\left(v X_{n}\right)\right]=h(v) .
$$

We deduce that there exists a measurable function $\tilde{h}$ on $G / H$ such that $\forall u \in U, h(u)=\widetilde{h}(\pi(u))$ and

$$
\forall u \in \pi(U), \quad \tilde{h}(u)=\int_{G / H} \tilde{h}(u g) \pi(\mu)(d g) .
$$

As $G / H$ is an abelian group, by taking again the arguments quoted in the introduction to obtain a generalization of the Choquet-Deny theorem in abelian semi-groups, we obtain, for all $n \geqslant 1$,

$$
u_{n}=\int_{G / H}\left(\tilde{h}\left(y_{1}+y_{2}+\cdots+y_{n}\right)-\tilde{h}\left(y_{2}+\cdots+y_{n}\right)\right)^{2} \mu\left(d y_{1}\right) \ldots \mu\left(d y_{n}\right)=0 .
$$

It follows that $\tilde{h}(\cdot)=\tilde{h}(e), \mu$-a.e., then $h(\cdot)=h(e), \mu$-a.e. and finally $\forall u \in T_{\mu}, h(u)=h(e)$. The application of this equality to the left translates $h^{g}: x \rightarrow h(g x)(g \in G)$ of $h$, gives us the required result.

3.3. Example. $G=\mathbb{R} \times \mathbb{R}^{d}$ with product $(a, x)(b, y)=\left(a+b, x+e^{a} y\right)$. Denote by $\pi$ the projection on $\mathbb{R}$. A pair $(G, \mu)$ acts on $\mathbb{R}^{d}$, in a recurrent way, if and only if the random walk $\pi\left(X_{n}\right)=\pi\left(Y_{1}\right)+\cdots+\pi\left(Y_{n}\right)$ is recurrent. This assumption is satisfied when $\mathbb{E}\left[\left|\pi\left(Y_{1}\right)\right|\right]<+\infty$ and $\mathbb{E}\left[\pi\left(Y_{1}\right)\right]=0$. If the random variables $\pi\left(Y_{1}\right)$ and $-\pi\left(Y_{1}\right)$ have the same distribution, this assumption is satisfied [8] if $\lim _{t \rightarrow+\infty} t \mathbb{P}\left[\left\{\pi\left(X_{1}\right)>t\right\}\right]=0$.

\section{References}

[1] R. Azencott, Espaces de Poisson des groupes localement compacts, in: Lecture Notes in Math., vol. 148, Springer, Berlin, 1970.

[2] A. Avez, Théorème de Choquet-Deny pour les groupes à croissance non exponentielle, CRAS Sér. A 279 (1974) 25-28.

[3] D. Blackwell, On transient Markov processes with a countable number of states and stationary transition probabilities, Ann. Math. Stat. 26 (1955) 654-658.

[4] G. Choquet, J. Deny, Sur l'équation de convolution $\mu=\mu * \sigma$, CRAS Sér. A 250 (1960) 799-801.

[5] Y. Derriennic, Entropie, théorèmes limite et marches aléatoires, in: Lecture Notes in Math., vol. 1210, Springer, Berlin, 1986, pp. $241-284$.

[6] E.B. Dynkin, M.B. Malyutov, Random walks on groups with a finite number of generators, Soviet Math. Dokl. 2 (1961) $399-402$.

[7] H. Federer, Geometric Measure Theory, Springer-Verlag, Berlin.

[8] H. Furstenberg, A Poisson formula for semi-simple Lie groups, Ann. Math., Ser. 277 (1963) 335-386.

[9] Y. Guivarc'h, Croissance polynomiale et périodes des fonctions harmoniques, Bull. Soc. Math. France 101 (1973) 333-379.

[10] W. Jaworski, Random walks on almost connected locally compact groups: boundary and convergence, J. Anal. Math. 74 (1998) $235-273$.

[11] V.A. Kaimanovich, A.M. Vershik, Random walks on discrete groups. Boundary and entropy, Ann. Probab. 11 (1983) 457-490.

[12] V.A. Kaimanovich, Poisson Boundaries of Random Walks on Discrete Solvable Groups, Plenum, New York, 1991, pp. 205-238.

[13] F. Ledrappier, Poisson boundaries of discrete groups of matrices, CRAS Sér. I 2978 (16) (1984) 393-396.

[14] J. Neveu, Bases mathématiques du calcul des probabilités, Masson, 1970.

[15] A. Raugi, Périodes des fonctions harmoniques bornées, Séminaire de Probabilités de Rennes, 1978.

[16] A. Raugi, Fonctions harmoniques et théorèmes limites pour les marches aléatoires sur les groupes, Bull. Soc. Math. France 54 (1977) $5-118$.

[17] D. Revuz, Markov Chains, North-Holland, Amsterdam, 1975. 